Gut and Liver, Vol. 10, No. 5, September 2016, pp. 672-686

REVIEW

\title{
Magnetic Resonance Elastography and Other Magnetic Resonance Imaging Techniques in Chronic Liver Disease: Current Status and Future Directions
}

\author{
Cher Heng $\operatorname{Tan}^{1}$ and Sudhakar Kundapur Venkatesh ${ }^{2}$ \\ ${ }^{1}$ Department of Diagnostic Radiology, Tan Tock Seng Hospital, Singapore, and ${ }^{2}$ Department of Radiology, Mayo Clinic, Rochester, MN, USA
}

Recent advances in the noninvasive imaging of chronic liver disease have led to improvements in diagnosis, particularly with magnetic resonance imaging (MRI). A comprehensive evaluation of the liver may be performed with the quantification of the degree of hepatic steatosis, liver iron concentration, and liver fibrosis. In addition, MRI of the liver may be used to identify complications of cirrhosis, including portal hypertension, ascites, and the development of hepatocellular carcinoma. In this review article, we discuss the state of the art techniques in liver MRI, namely, magnetic resonance elastography, hepatobiliary phase MRI, and liver fat and iron quantification MRI. The use of these advanced techniques in the management of chronic liver diseases, including nonalcoholic fatty liver disease, will be elaborated. (Gut Liver 2016;10:672-686)

Key Words: Magnetic resonance imaging; Magnetic resonance techniques; Liver cirrhosis; Liver steatosis; Non-alcoholic fatty liver disease

\section{BACKGROUND}

Recent advances in magnetic resonance imaging (MRI) of the liver have led to improvements in diagnosis of chronic liver diseases (CLDs) noninvasively. CLD, regardless of etiology, results in hepatocyte loss, inflammation, and then fibrosis, which would progress to cirrhosis of the liver if untreated. Liver fibrosis in early stages is reversible, and patients treated with early fibrosis have significantly higher survival rates as compared to those untreated. ${ }^{1}$ There is growing evidence that even cirrhosis may regress if appropriately treated. Detection and staging of liver fibrosis therefore has become important; however, until about the last decade, it depended on an invasive liver biopsy. Liver biopsy is limited by high cost, low patient acceptance, in- terobserver variability during microscopic evaluation, sampling error, poor reproducibility, and, importantly, an invasive nature with a complication rate of 3\% and a mortality rate of $0.03 \%{ }^{2,3}$ With the emergence of elastography techniques, the need for liver biopsy has rapidly diminished for diagnosis of clinically significant liver fibrosis.

With the availability of effective treatments for chronic viral hepatitis and the growing prevalence of obesity, nonalcoholic fatty liver disease (NAFLD) has now become the most common etiology for CLD. Understanding the pathogenesis of NAFLD for its effective treatment is of major research interest worldwide. This includes quantification of fat, detection of inflammation and fibrosis. Detection and quantification of fat and iron with MRI has almost eliminated the need for histological confirmation for NAFLD and iron overload states.

The introduction of hepatobiliary contrast agents that are actively taken up by hepatocytes gives an opportunity to assess liver function. These agents are proving to be valuable in the evaluation of functional reserve in patients undergoing major liver resections and for liver donors. In this paper, we shall discuss the technical aspects, strengths and limitations of MR elastography (MRE), hepatobiliary phase MRI, liver fat and liver iron quantification MRI.

\section{MR elastography}

MRE was introduced for clinical practice in 2007 and is now available at more than 600 sites worldwide. Currently, MRE is the most accurate noninvasive imaging technique for detection and staging of liver fibrosis. MRE has gained increasing popularity in recent years, in large part due to its higher technical success and ability to overcome some of the weaknesses of ultrasound-based methods for assessing liver fibrosis.

MRE of the liver is performed using an MRI safe passive driver that is applied to the right upper abdomen and lower

Correspondence to: Sudhakar Kundapur Venkatesh

Department of Radiology, Mayo Clinic, 200 First Street SW, Rochester, MN 55905, USA

Tel: +1-507-284-1728, Fax: +1-507-284-2405, E-mail: venkatesh.sudhakar@mayo.edu Received on October 1, 2015. Revised on November 29, 2015. Accepted on December 15, 2015. pISSN 1976-2283 eISSN 2005-1212 http://dx.doi.org/10.5009/gnl15492

@) This is an Open Access article distributed under the terms of the Creative Commons Attribution Non-Commercial License (http://creativecommons.org/licenses/by-nc/4.0) which permits unrestricted non-commercial use, distribution, and reproduction in any medium, provided the original work is properly cited. 
chest overlying the right lobe of the liver while the patient is being scanned in the MRI scanner (Fig. 1). Typically four 10$\mathrm{mm}$ slices are obtained through the largest cross-section of the liver. The MRE sequence is carried out with four short breathholds and completed within 1 to 2 minutes, without the need for intravenous contrast injection. An active driver generates low frequency mechanical waves (typically at $60 \mathrm{~Hz}$ ) which are conducted to the passive driver through a long plastic tube. The passive driver vibrates and produces shear waves that are propagated across the liver. The wavelength of the propagating shear wave is directly proportional to the stiffness of the liver, that is, the stiffer the liver, the longer the wavelength. To image these shear waves, a modified phase-contrast pulse sequence is used with cyclic motion encoding gradients that are synchronized to the mechanical waves. This allows generation of magnitude and phase images. By applying an inversion algorithm to the raw data, elastograms or stiffness maps (Fig. 2) that depict tis- sue stiffness are generated from the information from the wave images. ${ }^{4}$ Elastograms typically depict shear stiffness in units of kilopascals $(\mathrm{kPa})$, and may be displayed in a gray scale or with a color scale. ${ }^{5}$ A region of interest is drawn over the liver on the elastogram and a mean liver stiffness is calculated from the four slices. The tissue volume evaluated with four slices can be up to $1 / 3$ of the liver, and potentially the entire liver can be evaluated if a three-dimensional (3D) technique is used.

Reader-based methods of measurement require manual segmentation of liver tissue from the MR images, taking care to avoid nonhepatocyte tissues such as blood vessels. Hence it makes sense that the most reproducible method would be to average the readings of entire cross sections of the whole liver. ${ }^{6}$ Care must be taken to measure at same regions on follow-up cases and also to avoid potential areas of pitfalls which include the left lobe that is subject to cardiac pulsations, large vessels, liver edges, and so forth.

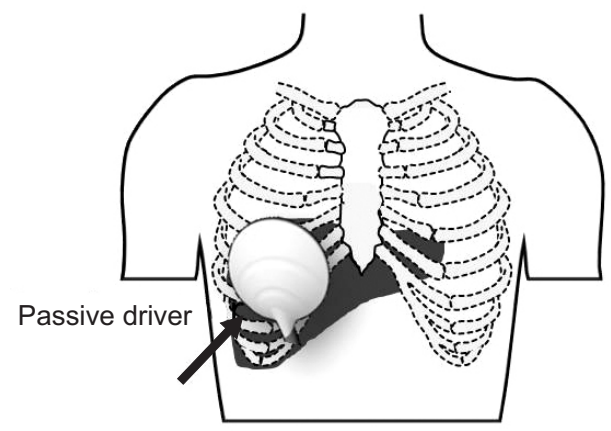

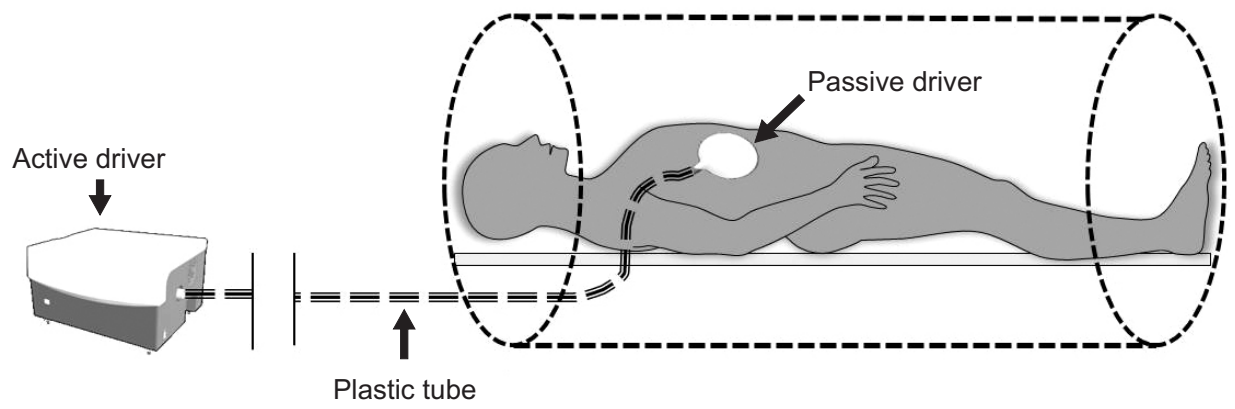

Plastic tube
Fig. 1. Diagram of the set-up used to perform clinical liver magnetic resonance elastography (MRE). MRE is performed with a subject in the supine position with a passive driver placed over the liver at the level of the xiphisternum. The passive driver is connected to the active driver placed outside the magnet room with a plastic tube.
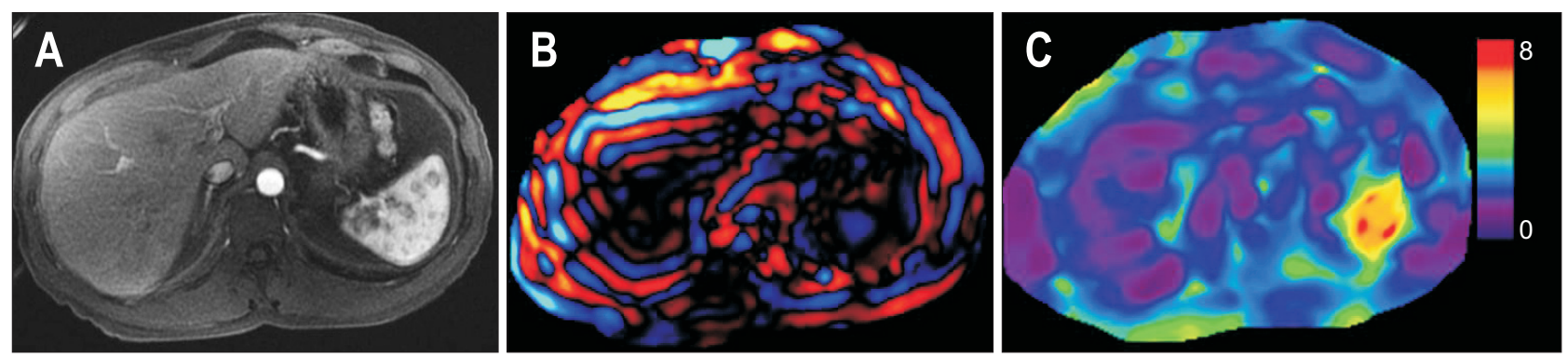

Fig. 2. Magnetic resonance elastography (MRE) of a normal liver. Anatomical image (A) indicates the cross-section through which MRE was performed. Post-processed wave image (B) indicates the propagation of shear waves through the cross-section of the liver, and the stiffness map (C) or elastogram indicates the spatial distribution of stiffness in color. A scale for interpretation is also provided; in the example, the scale is illustrated from 0 to $8 \mathrm{kPa}$. 

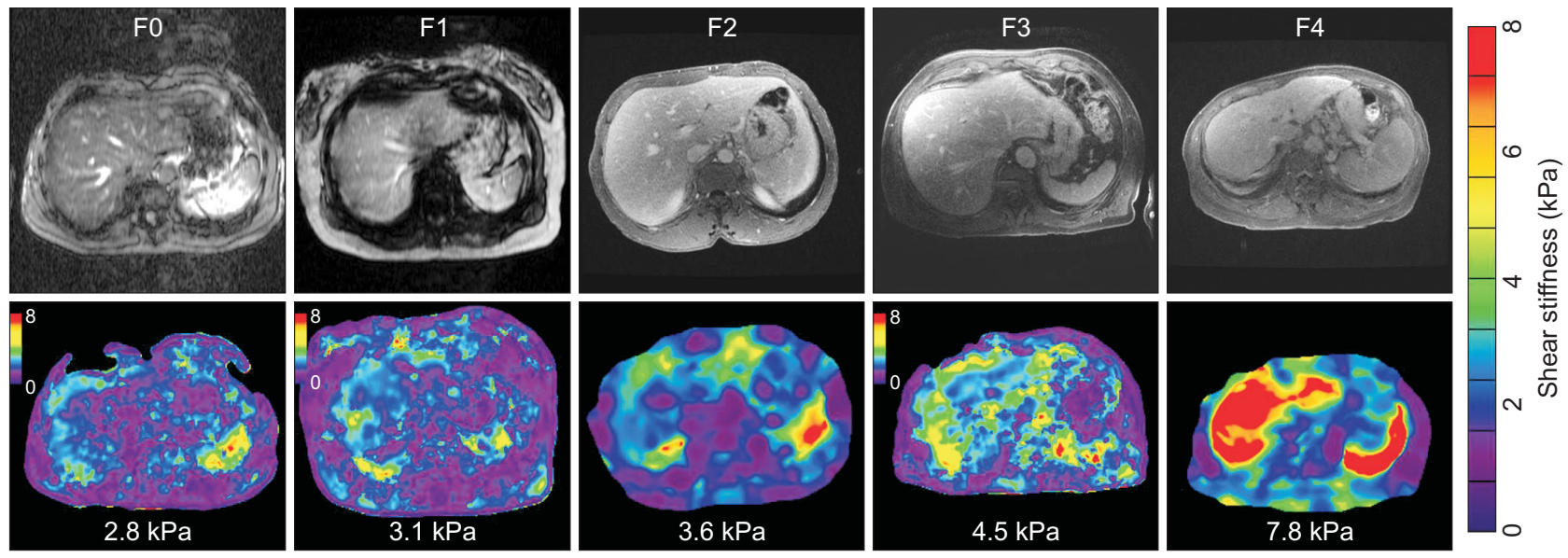

Fig. 3. Magnetic resonance elastography of the liver in chronic hepatitis $C$ patients with liver biopsy-proven fibrosis at METAVIR stages F0 through F4. Note the gradual increase in stiffness in the mild stages and incremental increase in the later stages of fibrosis.

Liver stiffness measured with MRE increases with increasing stage of fibrosis. The increase in stiffness, therefore differences between early stages of fibrosis is small (stage 0 vs stage 1 , stage 1 vs stage 2 ) and increases significantly with advanced fibrosis and cirrhosis (Fig. 3).

\section{1) Strengths of the technique}

The most significant advantage that MRE has over ultrasound-based methods of assessing liver fibrosis is its ability to depict tissue stiffness over the entire liver cross-section. As it is already widely known, fibrosis of the liver occurs in a heterogeneous fashion, particularly in the early stages of disease. This can result in sampling errors if only a portion of the liver is interrogated with techniques such as transient elastography (TE) or even percutaneous needle biopsy (commonly regarded as the reference standard for the diagnosis of liver fibrosis). With MRE, the distribution of stiffness can be demonstrated, while providing an opportunity for biopsy guidance. ${ }^{8}$

MRE has higher technical success rate than TE, ${ }^{9}$ and can be performed in obese patients, in patients where there is concomitant ascites or even in patients when there is colonic interposition between the liver and anterior abdominal wall. MRE can be performed before or after injection of MRI contrast agents and studies have shown no significant influence on measured liver stiffness, ${ }^{10,11}$ providing considerable flexibility of its use in the clinical liver MRI protocol.

The accuracy of MRE is better than routine serum liver function tests ${ }^{12,13}$ for detection of significant and advanced fibrosis. In the study by Huwart et al., ${ }^{9}$ the diagnostic accuracy of MRE (0.994 for $\mathrm{F}>2$, 0.985 for $\mathrm{F}>3$, and 0.998 for $\mathrm{F}>4$ ) was higher than TE, serum aspartate aminotransferase to platelets ratio index (APRI) and the combination of TE with APRI (0.837, 0.709 , and 0.849 for $\mathrm{F}>2 ; 0.906,0.816$, and 0.936 for $\mathrm{F}>3$; $0.930,0.820$, and 0.944 for $\mathrm{F}>4$, respectively). In the systematic review of 153 studies by Bonekamp et al., ${ }^{14}$ MRE was the only

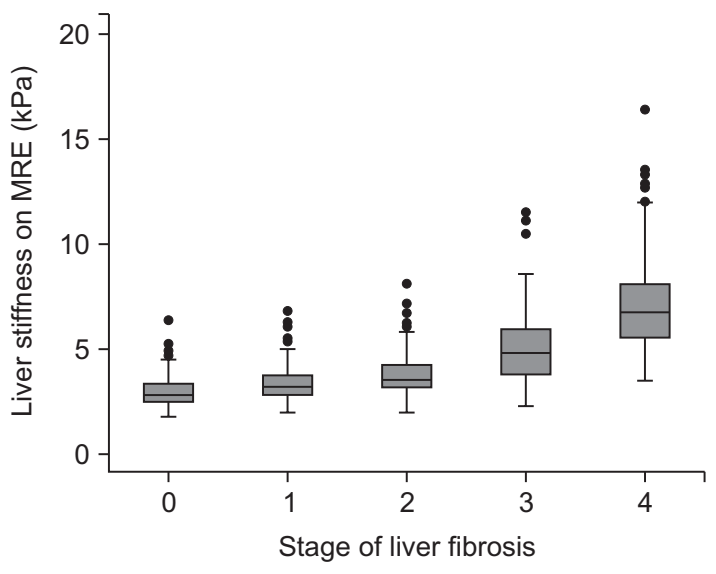

Fig. 4. Composite box plot graph indicates magnetic resonance elastography (MRE) and the stiffness values for various stages of fibrosis in 697 patients with different etiologies of chronic liver disease. The horizontal line through each box represents a median value, and each box top and box bottom represents data from the 25th to 75th percentiles, respectively (middle 50\% of 50\% of the observations). Whiskers represent the data from the minimum to maximum, excluding outliers, which are represented as separate dots. Adapted from Singh S, et al. Clin Gastroenterol Hepatol 2015;13:440-451.e6, with permission from Elsevier. ${ }^{19}$

noninvasive technique that was able to stage liver fibrosis or diagnose mild hepatic fibrosis with reasonable accuracy. In fact, MRE may predict for nonalcoholic steatohepatitis (NASH), even before the onset of liver fibrosis. ${ }^{15}$

Several more recent studies have attested to the ability of MRE to reliably differentiate between early and late stages of hepatic fibrosis. Compared to the standard anatomic evaluation of liver morphology, MRE performs significantly better for both significant fibrosis (0.989 in MRE vs 0.71 to 0.82 in MRI) and cirrhosis (0.935 in MRE vs 0.61 to 0.80 in MRI) ${ }^{16}$ Using a 3.7$\mathrm{kPa}$ cutoff value, the study by Venkatesh et al. ${ }^{6}$ found that MRE 
yielded a sensitivity of $91 \%$ and specificity of $80 \%$ for differentiating F0-F2 from F3-F4 grades of fibrosis. With a slightly lower threshold of $3.6 \mathrm{kPa}$ on MRE, Loomba et al. ${ }^{17}$ showed similar results (sensitivity, 86\%; specificity, 91\%) and an area under the receiver operating characteristic curve (AUROC) of 0.924 in NAFLD patients.

In a large retrospective study of 1,377 consecutive MRE examinations, Yin et al. ${ }^{18}$ from Mayo Clinic demonstrated a high technical success rate of 94.4\%; in addition the mean liver stiffness was significantly higher in patients with advanced fibrosis (stages F3 to F4) than in those with mild to moderate fibrosis (stages F0 to F2).
The findings from these various recent studies are concordant with a very recent meta-analysis by Singh et al. ${ }^{19}$ In the metaanalysis, 12 retrospective studies and 697 patients were included (Fig. 4). There was a relatively even distribution of patients in the various stages of fibrosis from F0 to F4. The AUROC for diagnosis of various stages of fibrosis using MRE ranged from 0.84 to 0.92 (Table 1). The authors found a high accuracy of the technique independent of body mass index and the etiology of CLD. $^{19}$

Being a quantitative technique, it is important that MRE has excellent intraobserver and interobserver reproducibility. This feature of MRE allows it to be used as a tool for monitoring dis-

Table 1. Diagnostic Performance of Magnetic Resonance Elastography for the Detection of Fibrosis in 697 Patients

\begin{tabular}{lcccccc}
\hline \multicolumn{1}{c}{ Fibrosis stage } & $\begin{array}{c}\text { Optimal cutoff } \\
\text { value, kPa }\end{array}$ & AUROC (95\% CI) & Sensitivity & Specificity & Positive LR & Negative LR \\
\hline Any fibrosis ( $\geq$ stage 1) & 3.45 & $0.84(0.76-0.92)$ & 0.73 & 0.79 & 3.48 & 0.34 \\
Significant fibrosis ( $\geq$ stage 2) & 3.66 & $0.88(0.84-0.91)$ & 0.79 & 0.81 & 4.16 & 0.26 \\
Advanced fibrosis ( $\geq$ stage 3) & 4.11 & $0.93(0.90-0.95)$ & 0.85 & 0.85 & 5.67 & 0.18 \\
Cirrhosis (stage 4) & 4.71 & $0.92(0.90-0.94)$ & 0.91 & 0.81 & 4.79 & 0.11 \\
\hline
\end{tabular}

Adapted from Singh S, et al. Clin Gastroenterol Hepatol 2015;13:440-451.e6, with permission from Elsevier. ${ }^{19}$ AUROC, area under the receiver operating characteristic curve; CI, confidence interval; LR, likelihood ratio.
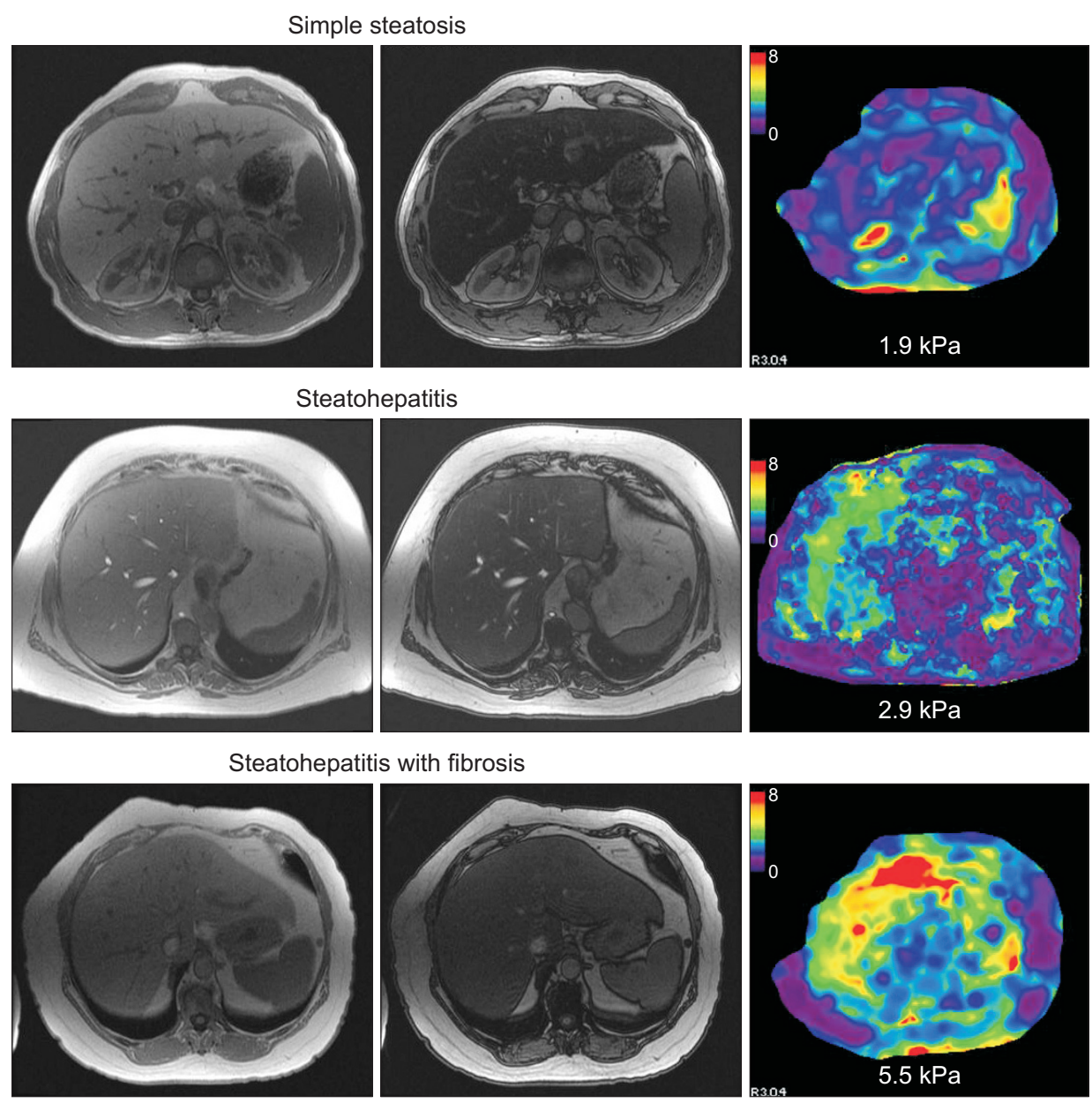

Fig. 5. Magnetic resonance elastography is useful for differentiating livers with simple steatosis (top row), which exhibits normal stiffness, from steatohepatitis (middle row), which exhibits a mild increase in stiffness, and steatohepatitis with fibrosis (bottom row), which exhibits a significant increase in stiffness. 
ease progression and for assessment of the treatment response. In the study by Shire et al. in patients with hepatitis C, MRE demonstrated high test-retest repeatability. ${ }^{20}$ MRE scanning using 3T scanners has also been found to be feasible. ${ }^{21,22}$ In a prospective study on 13 subjects, Serai et al. ${ }^{23}$ found that there was no significant difference in the results between scanners from two different vendors (Philips and General Electric). The study yielded a high interclass correlation coefficient value of 0.994 .

Perhaps the major role of MRE will be in the evaluation of NAFLD (Fig. 5). It has been shown with several studies that MRE can accurately differentiate simple steatosis from NASH and steatohepatitis with fibrosis. ${ }^{15,17,24}$ With the epidemic of obesity worldwide, MRE would be instrumental in the evaluation and assessment of treatment response in metabolic disorders. More discussion on this follows in the section on fat quantification.

\section{2) Limitations of the technique}

Currently, the main limitation of MRE is that it may fail in patients with moderate to severe iron overload secondary to hemochromatosis or hemosiderosis. In iron overload of the liver parenchyma, the liver MRI signal becomes too low and becomes undetectable with the standard MRE sequence, which is gradient-echo (GRE) based. ${ }^{25}$ However, newer MRI sequences have been developed that can assess liver stiffness in patients with mild to moderate iron deposition. ${ }^{26}$

With current MRE techniques, liver stiffness resulting from fibrosis and that from other conditions cannot be differentiated and this may lead to inaccurate results with MRE. Increased hepatic stiffness may be found in patients with acute liver inflammation even without fibrosis. ${ }^{27}$ When MRE is performed in patients with CLD in the postprandial state, increased portal blood flow results in a dynamic rise in liver stiffness, leading to overestimation of the grade of liver fibrosis. ${ }^{28}$ However, this can be easily overcome by having patients adequately fasted prior to the MRE examination and should be ideally scanned in the fasting status for follow up as well. It should be noted that no such significant differences are found in the case of normal healthy volunteers without any CLD. Acute biliary obstruction and passive congestion of the liver can also give rise to increased liver stiffness. MRE should be interpreted with caution when such conditions exist.

Chronic inflammation may have some effect on MRE evaluation of liver fibrosis. ${ }^{12,18,29}$ While some studies have shown that performance of MRE is affected by chronic inflammation, others have reported no significant effect. ${ }^{12,29-32}$

\section{3) MRE versus other elastography techniques}

Among the other various noninvasive methods of diagnosis of liver fibrosis, one-dimensional TE based on ultrasound (FibroScan; Echosens, Paris, France) is one of the most widely accepted and practiced modalities. ${ }^{33,34}$ TE with FibroScan has been widely validated for its ability to differentiate between the grades of fibrosis. ${ }^{34,35}$ The newer XL probe allows for high technical success and reliability compared to the standard M probe
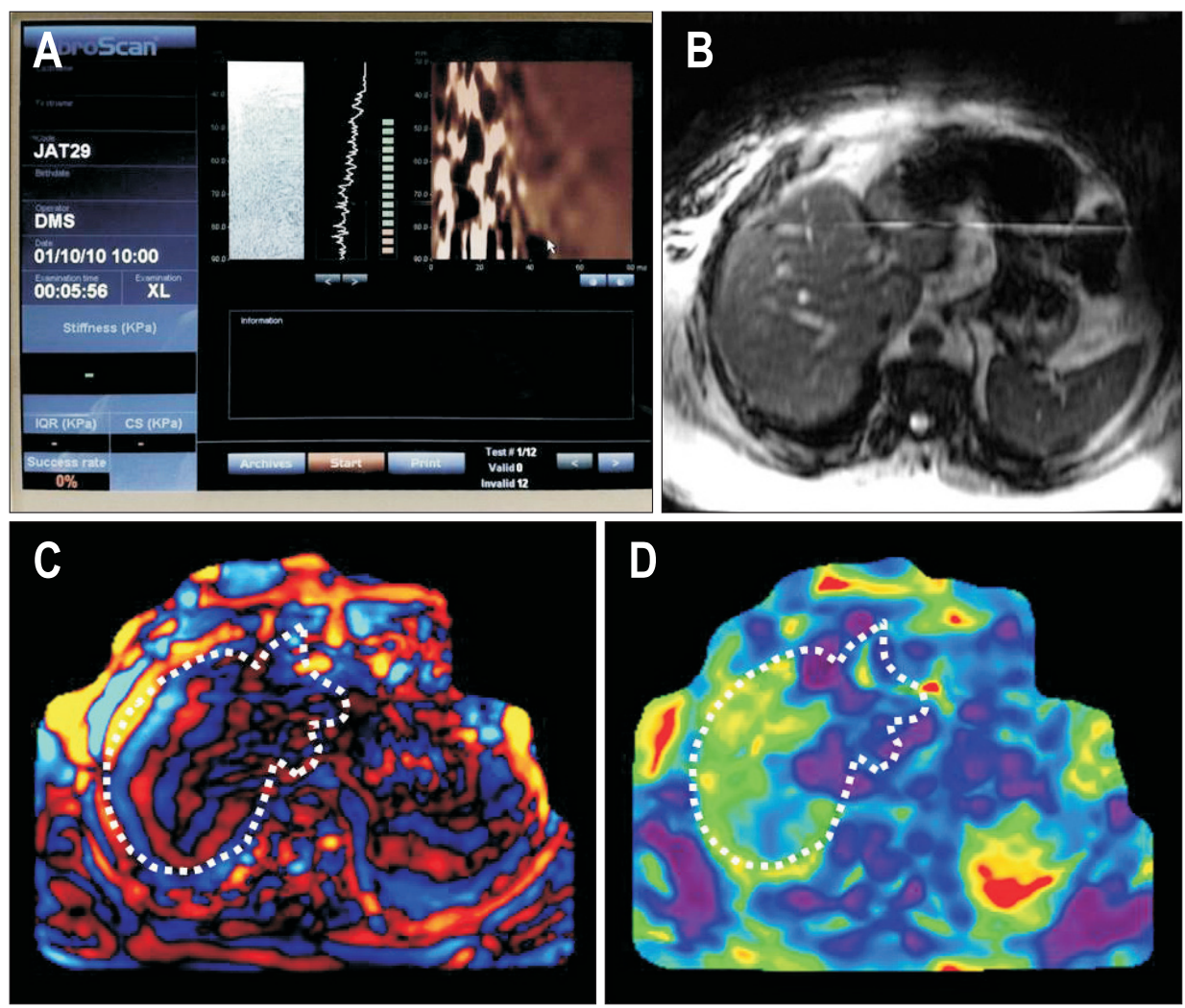

Fig. 6. An example of a failed measurement with transient elastography (TE) in an obese subject with a body mass index of $48 \mathrm{~kg} / \mathrm{m}^{2}$. A Fibroscan (Echosens) performed with a XL probe failed to provide valid measurements (A). Magnetic resonance elastography performed in the same patient was successful. Note thick subcutaneous fat in the magnitude image of MRE (B). MRE wave image (C) shows excellent demonstration of shear waves through the liver, as well as the spleen. The measured stiffness in this patient was $3.9 \mathrm{kPa}$ as shown on the stiffness map (D), which was consistent with mild liver fibrosis. A liver biopsy confirmed grade 1 steatohepatitis and stage 1 fibrosis. 
in obese patients. ${ }^{36}$

There are, however, a number of limitations with TE. Higher failure rates are encountered in patients with concomitant morbidities such as obesity (Fig. 6) and NASH. ${ }^{37}$ TE is also not useful for imaging patients with ascites, a common finding in CLD.

Furthermore, in FibroScan, the liver is interrogated without a corresponding grey-scale image on ultrasound, akin to a "blind" procedure. It is limited in that it can only measure a cylindrical volume that is $1-\mathrm{cm}$ wide and $4-\mathrm{cm}$ long, starting from $2.5-\mathrm{cm}$ deep to the skin surface, only in the right hepatic lobe and from a few intercostal spaces. ${ }^{38}$

Other ultrasound-based techniques include acoustic radiation force impulse imaging (ARFI), shear wave elastography and supersonic shear wave imaging (SSI). In these methods, the region of interest measurements (and elastograms in SSI) may be superimposed onto the anatomic grey-scale images. ${ }^{39}$ However, ARFI and SSI require more expertise on the part of the sonographer and are less well validated compared to TE.

\section{4) Future of MRE}

The scope of clinical applications of MRE is widening. The stiffness distribution can be diagnostic. For example, increased stiffness is present in a peripheral distribution in early primary sclerosing cholangitis and in the regions of congestion in congestive hepatopathy. The MRE technique is also undergoing several modifications and improvements. The spin-echo MRE technique has been developed for use in mild to moderate iron overload patients. The 3D MRE technique would be useful to estimate liver fibrosis burden and also focal liver lesions. A multifrequency MRE technique that can demonstrate changes in viscoelastic properties that can help separate inflammation, fibrosis and congestion is currently being developed.

MRE is useful in the evaluation of recurrence of fibrosis in liver transplants. ${ }^{8,40,41}$ MRE of the spleen and other organs in the abdomen can be performed. Simultaneous evaluation of liver and spleen stiffness is possible. Spleen stiffness correlates well with portal hypertension and is useful for prediction of significant varices. ${ }^{42-44}$ MRE is promising in the detection of hepatorenal syndrome. ${ }^{45}$

\section{Fat quantification by MRI}

\section{1) Background}

The importance of NAFLD as a cardiovascular and insulin resistance risk factor is widely known. Fatty liver is diagnosed when the intra-cytoplasmic fat deposition is found in greater than 5\% of hepatocytes. ${ }^{46,47}$ The histopathological method of diagnosis of hepatic steatosis is subject to large interobserver variability, since it requires the reader to visually determine the proportion of hepatocytes replaced by fat vacuoles. Histological analysis is a semi-quantitative method at best, and high rates of up to $24 \%$ of missed diagnoses may be encountered. ${ }^{48}$

With increasing recent interest in studying the effects of treatment on NAFLD, one may find that the traditional histologic method of categorizing steatosis into mild ( $>5 \%$ ), moderate (>30\%) and severe (>60\%) may not accurately reflect longitudinal changes of liver fat metabolism. Although currently there is no cutoff or limit where liver fat content is considered too much or harmful, it is foreseeable that as we understand this disease spectrum, there might arise a need to know the exact amount of fat in the liver for risk stratification or response assessment. Hence, there is a need for more precise quantitative methods of evaluating hepatic steatosis. Ultrasonography (US) and computed tomography (CT) can be used to diagnose hepatic steatosis. However, they are generally accurate only in the detection of moderate and severe steatosis, and are not able to reliably discriminate the degree of steatosis further. ${ }^{49,50}$ For the diagnosis of hepatic steatosis ( $\geq 30 \%$ fat content), US carries $60 \%$ to $65 \%$ sensitivity and 73\% to 77\% specificity. ${ }^{50,51}$ The accuracy of US tends to be reduced when cases of mild hepatic steatosis are included. ${ }^{49}$ Furthermore, since US evaluation of the degree of steatosis is based upon visual assessment, there exists higher degree of inconsistency within and between readers. Strauss and colleagues found that among 168 ultrasound examinations, the intra- and interobserver agreements were $54.7 \%$ to $67.9 \%$ and $47.0 \%$ to $63.7 \%$, respectively, when assessing the severity of hepatic steatosis using the traditional 4-point visual grading system. ${ }^{52}$ Although the Hounsfield attenuation of liver tissues can be measured, CT also cannot reliably quantify hepatic steatosis. In the study by van Werven et al., ${ }^{50} \mathrm{CT}$ yielded $74 \%$ sensitivity and 70\% specificity and an AUROC of 0.76, which was even lower than US. Dual energy CT scanning does not improve the accuracy of hepatic steatosis diagnosis over conventional single-energy CTs. ${ }^{53}$

In recent times, MRI has proven to be the imaging modality of choice for quantifying liver fat. In the study by Noureddin et al., ${ }^{54}$ MRI based methods of hepatic fat quantification were able to reflect much smaller changes in the degree of steatosis, and this correlated with the patients' body weights and serum alanine aminotransferase and aspartate aminotransferase levels. This makes MRI ideal as an imaging biomarker for assessing response to treatment of fatty liver. In fact, recent papers have primarily used MRI as a means to quantify hepatic fat when studying the effects of prognostication and treatment of hepatic steatosis in relation to NASH and impaired glucose tolerance. ${ }^{55-58}$

\section{2) Strengths of the technique}

MRI is regarded as the most accurate means of imaging assessment of hepatic steatosis. There are two primary methods of evaluation for estimation of hepatic fat fraction on MRI. The first is an imaging-based, chemical shift method (CSI) which takes advantage of the fact that protons (hydrogen atoms) in fat and water molecules are quite different in magnetic resonance properties and that the signal intensity of the liver at different time-points of image data acquisition (echo time) varies, 

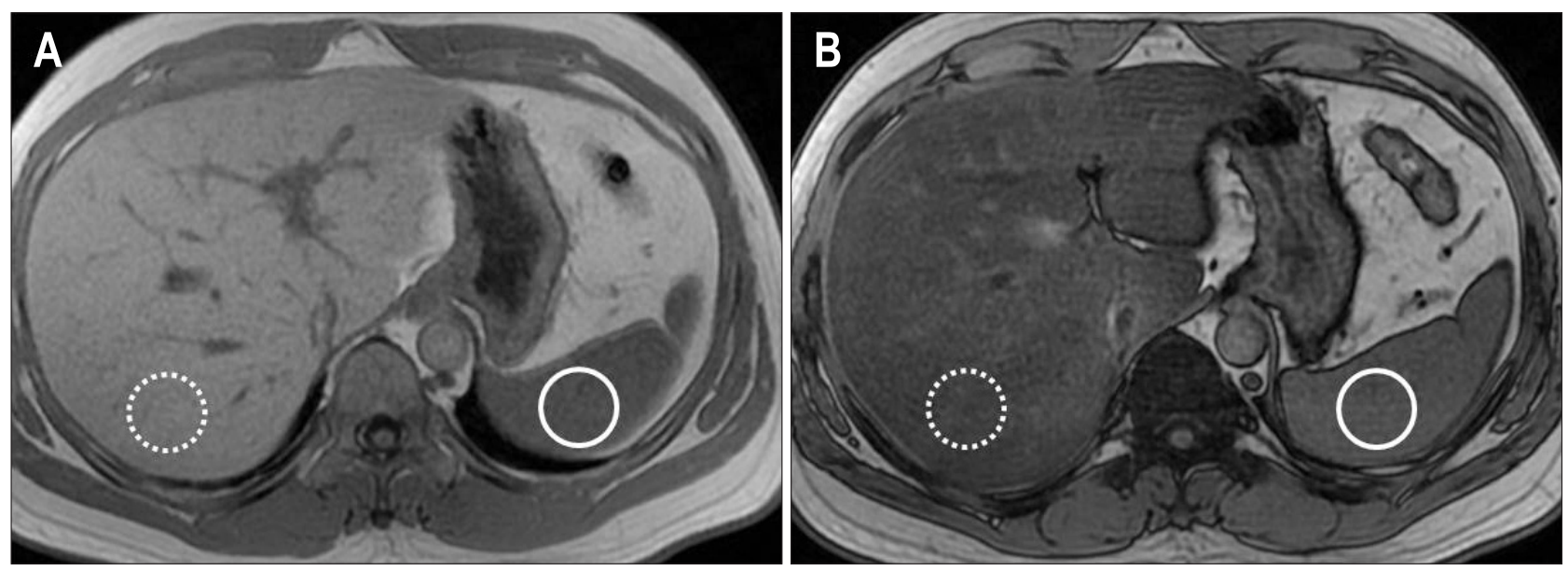

Fig. 7. Diffuse hepatic steatosis. (A) The in-phase image of the T1-weighted gradient recalled echo (dual echo) sequence indicates that the parenchymal signal intensity of the liver (dotted region of interest [ROI]) was greater than that of the spleen (black ROI). (B) The corresponding opposedphase image indicates that the parenchymal signal intensity of the liver was less than that of the spleen (solid ROI). Based on the liver-spleen signal intensity indices, the estimated fat fraction of the liver in this patient is $25.4 \%$.

depending on the concentration of water and fat (Fig. 7). This technique has a sensitivity and specificity of 90\% and 91\% respectively, using histopathology as the reference standard..$^{50}$ The second method is MR spectroscopy (MRS), a purely quantitative method that measures the concentration of water and fat metabolites based on their resonant frequencies. To avoid artifacts, MRS of the liver should ideally be performed within a single breath-hold. ${ }^{59}$ This can be achieved using single voxel imaging, in which a cubic volume sample of liver tissue is interrogated, typically up to $3 \times 3 \times 3 \mathrm{~cm}$ in dimensions. For accurate measurement, ensuring a homogeneous magnetic field across the region of interest using the technique of shimming is essential. ${ }^{60}$ Potentially, by combining the fat quantification information with MRE findings, one may be able to diagnosis simple hepatic steatosis from NASH and NASH with fibrosis.

\section{3) Limitations of the technique}

CSI for fat quantification is subject to the confounding effects of $\mathrm{T}^{*}$ (refers to decay of transverse magnetization caused by a combination of spin-spin relaxation and magnetic field inhomogeneitysignal decay and variations in T1-relaxation due to magnetic field inhomogeneity). In order to overcome this, low flip angle imaging (to overcome T1-related effects) and multiecho acquisitions (to correct for $\mathrm{T} 2 *$ decay) need to be performed. In doing so, fat quantification becomes more accurate, even in the presence of hepatic iron overload. ${ }^{59,61,62}$ Magnitudebased CSI is not able to determine fat fractions that are greater than 50\%. Taking into account phase information, iterative decomposition of water and fat using echo-asymmetry and the least-squares estimation (IDEAL) allows for hepatic fat fraction to be measured over the full dynamic range of hepatic steatosis up to $100 \% .^{63}$ Interestingly, even without sophisticated methods of correction, CSI is more accurate than CT or US. ${ }^{49}$ Since
MRS estimates hepatic fat fraction by directly measuring each fat and water peak, whilst CSI indirectly measures fat fraction by interrogating the magnitude of effects of signal interference between water and fat, MRS is widely regarded as the reference standard for imaging evaluation of hepatic fat fraction. With the aforementioned methods of $\mathrm{T} 2 *$ correction, coupled with fat spectral modeling and eddy current correction, excellent correlation is obtained between CSI and MRS. ${ }^{64,65}$ Furthermore, MRI measurements are highly reproducible across different magnet field strengths and machine vendors: the 95\% Bland-Altman limits-of-agreement between the 1.5T and 3.0T MRI scanners were approximately $2 \%$ to $4 \% .^{59}$ The main limitations for routine measurement of liver fat fraction in all patients include a requirement for technical expertise in scan acquisition and optimization. Advanced techniques may require additional postprocessing analysis.

\section{Liver iron quantification by MRI}

Iron overload leads to damage of hepatocytes and myocardium. The primary form of haemochromatosis is due to a recessive genetic disorder resulting in excessive iron being stored in both hepatocytes and Kupffer cells. The secondary form of haemochromatosis (hemosiderosis) is commonly due to excessive blood transfusions and blood disorders. Iron deposition in the secondary form is concentrated primarily in Kupffer cells of the reticuloendothelial system (RES).

Iron overload cannot be detected reliably on any other imaging technique except MRI. Iron is a paramagnetic substance and causes rapid decay of MRI signal and generally results in signal loss where it is found abundantly, such as the RES in organs such as liver, spleen and bone marrow. In the primary form of the disease, iron deposition can be seen in non-RES organs, notably in the pancreas. In severe cases of primary haemochro- 

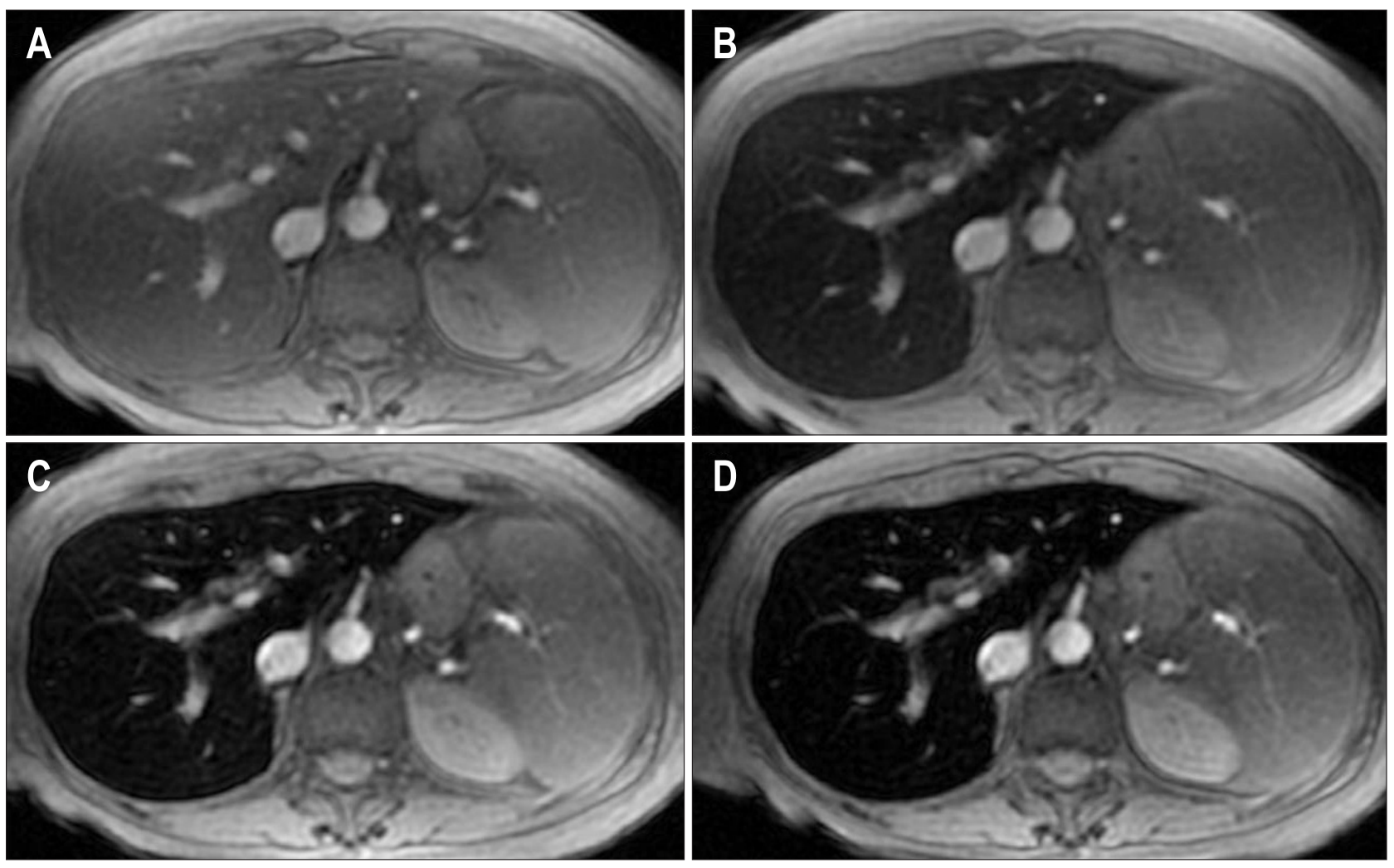

Fig. 8. Hepatic iron overload. There is a significant loss of the signal intensity with an increasing echo time (TE) from $1.536 \mathrm{msec}$ in (A) to 3.88 msec in (B) to $8.568 \mathrm{msec}$ in (C) to $10.912 \mathrm{msec}$ in (D). Using the T2* relaxometry method, the estimated concentration of iron per dry weight of liver tissue was $10 \mathrm{mg} / \mathrm{g}$, which is consistent with a moderately severe degree of hepatic iron overload.
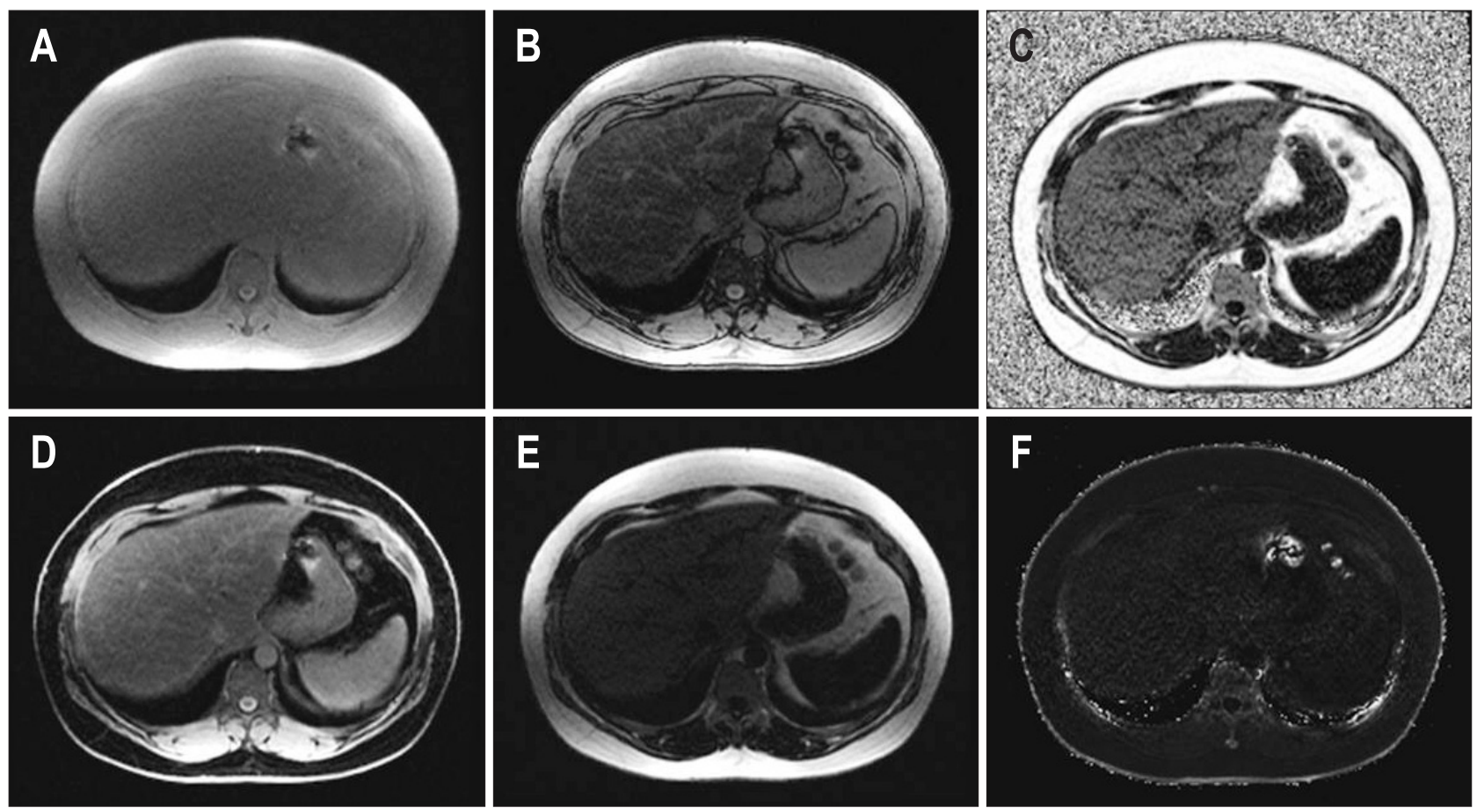

Fig. 9. Magnetic resonance imaging of a healthy volunteer with a fatty liver performed with the iterative decomposition of water and fat with echo asymmetry and least-squares estimation (IDEAL) sequence. The sequence provides in-phase (A), opposed-phase (B), fat signal fraction (C), water-only image (D), fat-only image, (E) and $\mathrm{R} 2^{*}$ maps (F). The fat and iron content in the liver can be calculated with the fat signal fraction map and R2* map, respectively. Note that the liver has a lower signal intensity compared with the spleen in the opposed-phase and water images and a higher signal intensity compared with the spleen in the fat image and fat fraction map. 
matosis, phlebotomy reduces the iron stores in the body, while in severe cases of secondary haemochromatosis, iron-chelating agents are used for treatment. ${ }^{66}$ Quantification of the degree of iron deposition is important for two reasons: it influences the decision to treat and provides an objective means to monitor response to treatment. In patients with chronic hepatitis C, liver iron overload is associated with disease progression and resistance to antiviral therapy. ${ }^{67}$

To quantify the degree of iron deposition, indirect markers such as serum ferritin and transferrin saturation have been used. Unfortunately, these tests are sensitive but are not specific. ${ }^{68}$ Random percutaneous needle biopsy of liver tissues with direct histological visualization of Prussian blue stained iron granule is the current gold standard to diagnose excessive hepatic iron deposition. The most common method of diagnosis is a semiquantitative method devised by Rowe et al. ${ }^{69}$ Atomic absorption spectrophotometry for direct hepatic iron quantification is not widely available and is limited by the fact that the biopsy specimen is destroyed during analysis and can no longer be evaluated histologically. ${ }^{70}$ Percutaneous needle biopsy suffers from sampling error, with a high coefficient of variation from 19\% in a healthy liver to $40 \%$ in the case of cirrhosis. ${ }^{71}$

MRI can be used to estimate the degree of iron deposition in tissues by virtue of the fact that iron accelerates $\mathrm{T} 2$ and $\mathrm{T} 2{ }^{*}$ signal decays in spin-echo and GRE pulse sequences respectively (Fig. 8). The effects of iron on signal loss are relatively greater with GRE pulse sequences and longer echo times. While GRE sequences may be more sensitive to low iron content, they may also suffer from inaccuracies in cases of severe iron deposition. ${ }^{72}$ In order to guide treatment initiation and therapy monitoring, a quantitative means of predicting the liver iron concentration (LIC) is necessary. ${ }^{73}$

It is also possible to evaluate fat signal fraction and LIC using a Dixon type sequence that produces fat signal fraction maps and R2* maps in one single method (Fig. 9). The technique provides correction for presence of iron and fat when measuring fat signal fraction and LIC, respectively.

\section{1) Strengths and limitations of the technique}

There are two main methods of assessing LIC on MRI: relaxometry measuring $\mathrm{T} 2$ or $\mathrm{T} 2{ }^{*}$ decay, and signal intensity comparisons between the liver and paraspinal muscles. ${ }^{74}$ As with other quantitative techniques, the prerequisite is a method that is accurate, reproducible and can be standardized across the world.

Using the relaxometry technique, $\mathrm{T} 2$ and $\mathrm{T} 2{ }^{*}$ values have shown strong correlation with LIC measured by liver biopsies. ${ }^{75}$ While high liver iron levels certainly portend a poorer prognosis, it is important to note that low or normal liver iron stores do not exclude iron overload in the myocardium or other specific target tissues ${ }^{76}$ that may similarly convey poorer prognosis. The main advantage of the relaxometry technique is that they also allow for myocardial iron quantification. Unfortunately, these techniques require specialized software and are not widely available.

The signal intensity ratio (SIR) method relies on GRE sequences due to their greater sensitivity to iron, compared with spin-echo sequences. The method developed by Gandon et al. ${ }^{77}$ from the University of Rennes in France is the most widely recognized SIR method. First, MR images using five GRE sequences that are each acquired in a single breath-hold and of a different echo time and flip angle are obtained. On each image, signal intensity readings of regions of interest in the right hepatic lobe and the right and left paraspinal muscles are obtained. By comparing the SIRs of liver to paraspinal muscles on a nomogram, reasonably accurate estimation of hepatic iron stores may be obtained. However, the SIR method is unable to further quantify LIC that exceeds $350 \mu \mathrm{mol} \mathrm{Fe/g.} \mathrm{Furthermore,} \mathrm{it} \mathrm{tends} \mathrm{to} \mathrm{overes-}$ timate LIC. ${ }^{78}$ Therefore, the utility of the LIC methods for clinical purpose is somewhat limited.

\section{Hepatobiliary phase MRI}

In patients with CLD, particularly those who require major liver resections, assessment of hepatic function is critical. Current methods of clinical evaluation, such as the Child-Pugh and Model for End-stage Liver Disease (MELD) scoring systems, provide a global assessment of hepatic function. In planning for major liver resection and transplantation, however, knowing the regional liver function of the proposed future liver remnant becomes important.

As such, there has recently been growing interest in the use of MRI for regional evaluation of liver function. This has been spurred by the advent of hepatocyte specific contrast. The first commercially available hepatocyte specific contrast was manganese based. This did not become popular for two reasons: (1) hepatocyte specific phase scan could only be performed a few hours after contrast administration, reducing the throughput for MRI scanners and (2) dynamic multiphase imaging cannot be performed. Subsequent commercially available hepatocyte specific contrast agents were gadolinium-based agents, allowing for standard multiphasic liver acquisitions before hepatocyte specific phase acquisition. These agents provide additional value to the diagnostic examinations.

With gadoxetic acid (Gd-EOB-DTPA, Gadoxetate), due to its high rate of excretion by the hepatocytes into the biliary tree, the hepatocyte specific phase occurs approximately 15 to 20 minutes post contrast administration, significantly improving the throughput for clinical liver MRI studies. In early animal testing, gadoxetate was shown to be transported by the organic anion transporter (OAT), ${ }^{79}$ and ischemia-induced damaged regions of the liver displayed significantly different enhancement compared to normal regions of the liver. ${ }^{80,81}$ Following transport across OAT into hepatocytes, gadoxetate is then excreted unchanged directly into the biliary tree via the ATP-dependent 

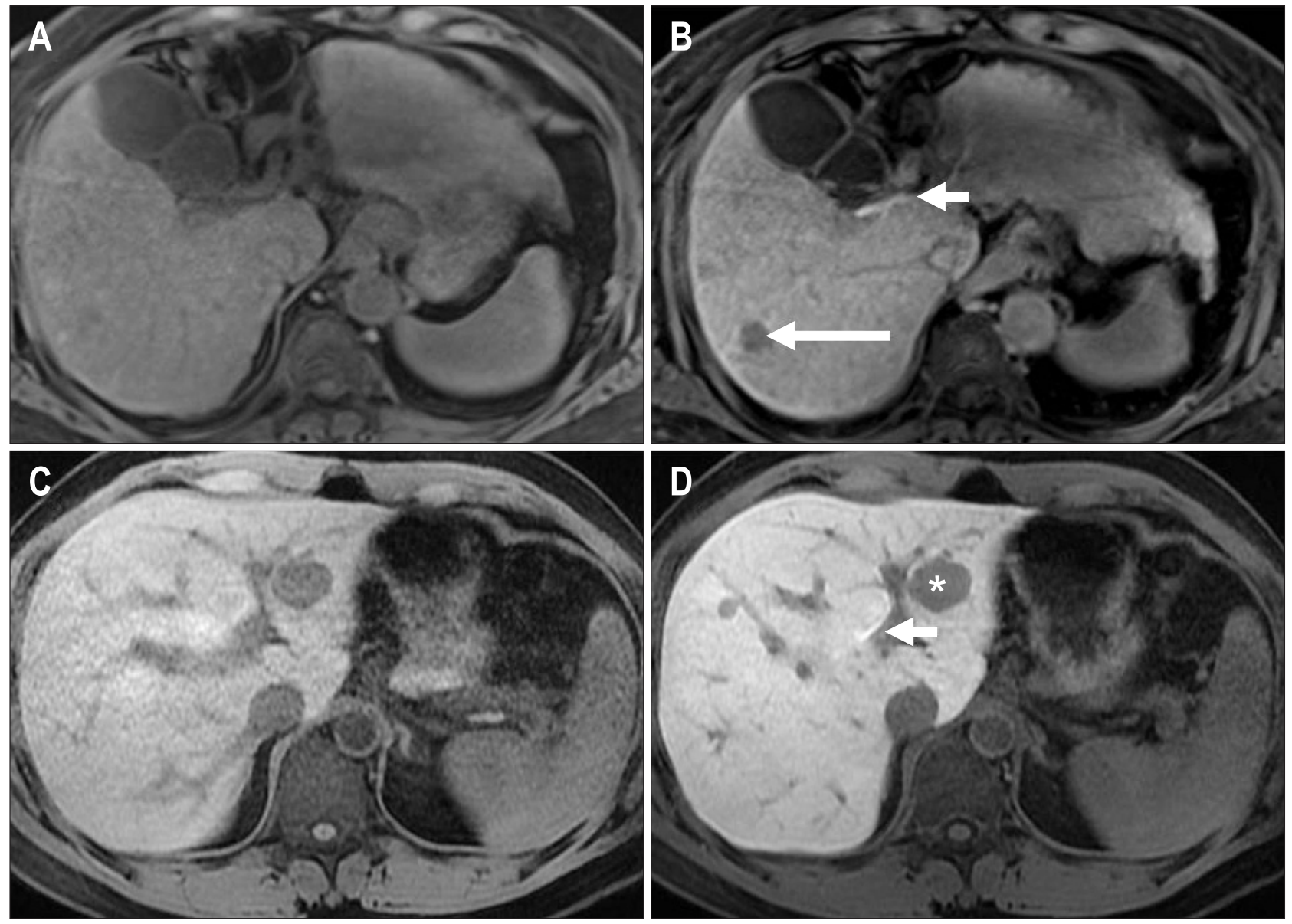

Fig. 10. Gd-EOB-DTPA indicates functional differences between cirrhotic and normal livers. The cirrhotic liver (A) exhibits a minimal increase in the signal intensity in the post-contrast T1-weighted image (B) at 20 minutes in the hepatobiliary phase. Note the presence of a small hepatocellular carcinoma in segment 7 (arrows) in (B). In contrast, in the normal liver (C), there is a significant increase in the signal intensity in the postcontrast VIBE image (D) at 20 minutes in the hepatobiliary phase compared with the pre-contrast VIBE image (A). There was an incidental hepatic hemangioma in the lateral segment of the left lobe (asterisk). Note the presence of contrast material excreted into the biliary ducts (short arrow) in both livers.

canalicular membrane multidrug resistance protein 2 (MRP2). ${ }^{82}$ Tsuda et al. ${ }^{83}$ further showed that it may be possible to differentiate NASH from hepatic steatosis using the relative enhancement method.

\section{1) Strengths of the technique}

Initial studies have alluded to gadoxetate enhanced MRI being a promising method for assessing regional liver function. In this method, the change in $\mathrm{T} 1$ relaxation times, and consequently the enhancement on MRI, reflects the concentration of gadolinium that is accumulated in normal hepatocytes via OAT peptides (OATP). ${ }^{84}$ As such, regions of the liver where hepatocyte OATP function is impaired will tend to accumulate less gadolinium than areas where hepatocyte function is normal, during the hepatocyte specific phase (Fig. 10). This translates to shortening of the T1 relaxation time by gadolinium and consequently lower signal intensity. In a recent study by Verloh et al., ${ }^{85}$ in which there were 150 patients scanned on a 3T MRI scanner, the use of gadoxetic acid enhanced MRI carried a sen- sitivity of 82.8\%, specificity of $92.7 \%$ and an AUROC of 0.87 for establishing differentiation between those with a normal liver function (MELD score $\leq 10$ ) and those with impaired liver function (MELD score $>10$ ).

In an early study by Nilsson et al ${ }^{86}$ where 12 patients with primary biliary cirrhosis were compared with 20 healthy volunteers, the patients with PBC displayed lower hepatocyte extraction fraction compared with controls; these differences increased with increasing MELD and Child-Pugh scores. Higaki et al. ${ }^{87}$ further showed that the degree of relative enhancement was reduced in patients with CLD with elevated bilirubin and aspartate aminotransferase levels. Nakamura et al. ${ }^{88}$ showed that serum albumin levels and prothrombin times predicted liver enhancement in the hepatobiliary phase. Utsunomiya et al ${ }^{89}$ were also able to demonstrate a high correlation between relative signal intensities and liver function tests, including the indocyanine green excretion test, which is commonly used in many centers in Asia for preoperative assessment of hepatic reserve. ${ }^{89}$

The main advantage of MRI-based evaluation over other con- 
ventional methods of clinical assessment is its ability to depict regional hepatic function. In the study by Nilsson et al., ${ }^{90}$ it has been shown that global liver function assessment may overestimate the function of the remnant liver segment in nine out of 10 cirrhotic patients by up to $9 \%$.

\section{2) Limitations of the technique}

As this method is relatively new, it requires more evidence to validate its use to assess regional liver function. Importantly, there is no consensus on the technique of quantifying the concentration of gadolinium in the liver tissues. The simpler method in practice will be the relative enhancement method, where the signal intensities of the liver tissues are compared before and after (during hepatocyte specific phase) contrast administration, akin to the measurements of signal intensities in the in-phase and opposed-phase in fat quantification. However, this method is subject to magnetic field inhomogeneity, whereby areas which are subject to a smaller magnetic moment may display reduced signal intensities.

Magnetic field inhomogeneity is inevitable in clinical scanning and these would reduce the accuracy of the relative enhancement method. In the study by Kanki et al., ${ }^{91}$ which studied 62 patients with chronic hepatitis or cirrhosis using a relative enhancement technique, there was no significant correlation between the MR grading of morphologic severity and the relative enhancement of liver tissues during hepatocyte specific phase.

Hepatic parenchymal enhancement also does not decrease according to the severity of morphologic changes in cirrhosis, leading to the hypothesis that signal intensity differences are more dependent on the hepatocyte function rather than the severity of morphologic changes in cirrhosis. However, in the study by Goshima et al., relative enhancement of the liver appeared to correlate with the degree of fibrosis, although in that study, MRI evaluation was studied using linear regression technique combining relative enhancement with liver-to-spleen volumetric ratio, to yield a sensitivity of $100 \%$, specificity of 73\% and an AUROC of 0.91. The more technically demanding method, which requires acquisition of more than one flip angle during scanning, thereby increasing scan times, is scientifically closer to measuring the actual concentration of gadolinium in liver tissues, as it removes the effects of magnetic field inhomogeneity. Katsube et al. ${ }^{92}$ were able to show that the T1 relaxation times were significantly longer in Child-Pugh B cirrhosis patients compared with Child-Pugh A cirrhosis patients and in both groups compared with patients with normal liver function. This may be attributed to the upregulation of the MRP2 transporter that excretes gadoxetic acid into the bile canaliculi. However, false negative results can be seen in patients who suffer from moderate hepatic fibrosis (stage F1 or F2) and necroinflammation, when the MRP2 transporters are normal or reduced respectively. ${ }^{93}$

Due to the fact that gadoxetic acid requires the presence of
OAT (specifically OATP1B1 and OATP1B3), false positive results may ensue if a broad number of compounds and competition for uptake may limit its uptake by hepatocytes. An example would be the antituberculous drug, Rifampicin. ${ }^{94}$ Furthermore, it has been shown that liver enhancement by gadoxetic acid in healthy subjects can be reduced by approximately 30\% to $40 \%$ due to genetic polymorphisms of the OATP1B1 transporter. ${ }^{95}$

\section{OTHER TECHNIQUES}

Diffusion weighted imaging (DWI) may be useful for detection of advanced fibrosis and cirrhosis. However, DWI suffers from lack of standardization across platforms and lower accuracy, as the apparent diffusion coefficient values overlap widely between normal liver parenchyma and fibrotic or cirrhotic liver. ${ }^{96}$

Perfusion imaging is promising for evaluation of liver fibrosis. However, this technique needs considerable post processing and is currently available only in advanced centers. At the present time, this technique is not used clinically.

Other MRI-based techniques including fractional extracellular space estimation $^{97}$ and magnetization transfer ratio imaging have not been proven to be useful for distinguishing healthy from cirrhotic liver. ${ }^{98}$

Since T1 $\rho$ (spin-lattice relaxation time in the rotating frame) MR imaging has been proposed to be sensitive to the macromolecular composition of tissues, it is plausible that whole liver T1 $\rho$ MRI may be sensitive for evaluation of liver fibrosis. Initial studies have shown that the mean T1-p values in fibrotic livers are higher than those in healthy patients, and this may provide differentiation. ${ }^{99,100}$ Strong evidence is still lacking, and this technique requires validation. Technique challenges encountered with T1 $\rho$ MRI include an increased sensitivity to B0 and B1 field inhomogeneities and a high specific absorption rate because of increased radiofrequency usage. ${ }^{101}$ Susceptibility weighted imaging is also considered feasible to detect moderate and advanced liver fibrosis, but further studies are needed to evaluate its clinical utility. ${ }^{102}$

\section{FUTURE DIRECTIONS}

There is increasing interest in the role of MRE for noninvasive diagnosis of NASH. MRE findings are not affected by steatosis. In fact, the study by Yoon et al. ${ }^{103}$ showed that MR-based fat quantification and MRE can be performed at the same sitting, yielding AUROC of 0.93 for fat quantification and 0.85 for MRE in living liver donor candidates. At present MRE suffers from lower spatial resolution, especially compared to standard anatomic MR images of the liver. More sophisticated inversion algorithms may allow for 3D depiction of liver stiffness, thereby improving spatial resolution and accuracy. Advancements in image acquisition technology would reduce scan time and minimize respiratory motion artifacts. Instead of using conventional 
gradient echo pulse sequences for image acquisition, spin echo, fast spin echo and echo planar imaging methods may increase signal to noise ratios, potentially reducing the limitation of hepatic iron overload. By applying more elaborate mechanical models to process the information obtained from wave images, we may be able to study additional tissue characteristics, such as attenuation, anisotropy, and nonlinearity. This may allow MRE to go beyond the current role of determining liver stiffness and perhaps allow it to discriminate between the effects of inflammation, passive congestion, fibrosis, and scarring. ${ }^{104}$

Combining MRE with gadoxetate imaging may help in assessing functional reserve and its correlation with CLD. In the future a comprehensive MRI study that would provide quantification of fibrosis, fat content and iron overload ("Hepatogram") may be available.

\section{CONCLUSION}

Recent advances in the imaging of liver disease has led to improved diagnosis of liver diseases. In this paper, we have discussed, in depth, the role of various MRI techniques, their strengths and limitations, as well as the potential roles of these techniques for improving diagnosis.

\section{CONFLICTS OF INTEREST}

No potential conflict of interest relevant to this article was reported.

\section{REFERENCES}

1. Jézéquelemail C, Bardou-Jacquet E, Desille Y, et al. P0709: survival of patients infected by chronic hepatitis $\mathrm{C}$ and FOF1 fibrosis at baseline after a 15 year follow-up. J Hepatol 2015;62(Suppl 2):S589.

2. Maharaj B, Maharaj RJ, Leary WP, et al. Sampling variability and its influence on the diagnostic yield of percutaneous needle biopsy of the liver. Lancet 1986;1:523-525.

3. Piccinino F, Sagnelli E, Pasquale G, Giusti G. Complications following percutaneous liver biopsy: a multicentre retrospective study on 68,276 biopsies. J Hepatol 1986;2:165-173.

4. Venkatesh SK, Yin M, Ehman RL. Magnetic resonance elastography of liver: technique, analysis, and clinical applications. J Magn Reson Imaging 2013;37:544-555.

5. Manduca A, Lake DS, Kruse SA, Ehman RL. Spatio-temporal directional filtering for improved inversion of MR elastography images. Med Image Anal 2003;7:465-473.

6. Rusak G, Zawada E, Lemanowicz A, Serafin Z. Whole-organ and segmental stiffness measured with liver magnetic resonance elastography in healthy adults: significance of the region of interest. Abdom Imaging 2015;40:776-782.

7. Marcellin P, Asselah T, Boyer N. Fibrosis and disease progression in hepatitis C. Hepatology 2002;36(5 Suppl 1):S47-S56.

8. Lee VS, Miller FH, Omary RA, et al. Magnetic resonance elastography and biomarkers to assess fibrosis from recurrent hepatitis $\mathrm{C}$ in liver transplant recipients. Transplantation 2011;92:581-586.

9. Huwart L, Sempoux C, Vicaut E, et al. Magnetic resonance elastography for the noninvasive staging of liver fibrosis. Gastroenterology 2008;135:32-40.

10. Hallinan JT, Alsaif HS, Wee A, Venkatesh SK. Magnetic resonance elastography of liver: influence of intravenous gadolinium administration on measured liver stiffness. Abdom Imaging 2015;40:783-788.

11. Motosugi U, Ichikawa T, Sou H, et al. Effects of gadoxetic acid on liver elasticity measurement by using magnetic resonance elastography. Magn Reson Imaging 2012;30:128-132.

12. Venkatesh SK, Wang G, Lim SG, Wee A. Magnetic resonance elastography for the detection and staging of liver fibrosis in chronic hepatitis B. Eur Radiol 2014;24:70-78.

13. Huwart L, Sempoux C, Salameh N, et al. Liver fibrosis: noninvasive assessment with MR elastography versus aspartate aminotransferase-to-platelet ratio index. Radiology 2007;245:458-466.

14. Bonekamp S, Kamel I, Solga S, Clark J. Can imaging modalities diagnose and stage hepatic fibrosis and cirrhosis accurately? J Hepatol 2009;50:17-35.

15. Chen J, Talwalkar JA, Yin M, Glaser KJ, Sanderson SO, Ehman RL. Early detection of nonalcoholic steatohepatitis in patients with nonalcoholic fatty liver disease by using MR elastography. Radiology 2011;259:749-756.

16. Venkatesh SK, Yin M, Takahashi N, Glockner JF, Talwalkar JA, Ehman RL. Non-invasive detection of liver fibrosis: MR imaging features vs. MR elastography. Abdom Imaging 2015;40:766-775.

17. Loomba R, Wolfson T, Ang B, et al. Magnetic resonance elastography predicts advanced fibrosis in patients with nonalcoholic fatty liver disease: a prospective study. Hepatology 2014;60:19201928.

18. Yin M, Glaser KJ, Talwalkar JA, Chen J, Manduca A, Ehman RL. Hepatic MR elastography: clinical performance in a series of 1377 consecutive examinations. Radiology 2015;278:114-124.

19. Singh S, Venkatesh SK, Wang Z, et al. Diagnostic performance of magnetic resonance elastography in staging liver fibrosis: a systematic review and meta-analysis of individual participant data. Clin Gastroenterol Hepatol 2015;13:440-451.e6.

20. Shire NJ, Yin M, Chen J, et al. Test-retest repeatability of MR elastography for noninvasive liver fibrosis assessment in hepatitis C. J Magn Reson Imaging 2011;34:947-955.

21. Park HS, Kim YJ, Yu MH, Choe WH, Jung SI, Jeon HJ. ThreeTesla magnetic resonance elastography for hepatic fibrosis: comparison with diffusion-weighted imaging and gadoxetic acidenhanced magnetic resonance imaging. World J Gastroenterol 2014;20:17558-17567.

22. Mannelli L, Godfrey E, Graves MJ, et al. Magnetic resonance elastography: feasibility of liver stiffness measurements in healthy volunteers at 3T. Clin Radiol 2012;67:258-262. 
23. Serai SD, Yin M, Wang H, Ehman RL, Podberesky DJ. Cross-vendor validation of liver magnetic resonance elastography. Abdom Imaging 2015;40:789-794.

24. Kim D, Kim WR, Talwalkar JA, Kim HJ, Ehman RL. Advanced fibrosis in nonalcoholic fatty liver disease: noninvasive assessment with MR elastography. Radiology 2013;268:411-419.

25. Taouli B, Ehman RL, Reeder SB. Advanced MRI methods for assessment of chronic liver disease. AJR Am J Roentgenol 2009;193:14-27.

26. Mariappan YK, Venkatesh SK, Glaser KJ, McGee KP, Ehman RL. MR elastography of liver with iron overload: development, evaluation and preliminary clinical experience with improved spin echo and spin echo EPI sequences. Proceedings of the 21st Annual ISMRM Conference (abstract 985); 2013 Apr 20-26; Salt Lake City, USA.

27. Arena U, Vizzutti F, Corti G, et al. Acute viral hepatitis increases liver stiffness values measured by transient elastography. Hepatology 2008;47:380-384.

28. Yin M, Talwalkar JA, Glaser KJ, et al. Dynamic postprandial hepatic stiffness augmentation assessed with MR elastography in patients with chronic liver disease. AJR Am J Roentgenol 2011;197:64-70.

29. Ichikawa S, Motosugi U, Nakazawa T, et al. Hepatitis activity should be considered a confounder of liver stiffness measured with MR elastography. J Magn Reson Imaging 2015;41:12031208.

30. Venkatesh SK, Xu S, Tai D, Yu H, Wee A. Correlation of MR elastography with morphometric quantification of liver fibrosis (Fibro-C-Index) in chronic hepatitis B. Magn Reson Med 2014;72:1123-1129.

31. Ichikawa S, Motosugi U, Ichikawa T, et al. Magnetic resonance elastography for staging liver fibrosis in chronic hepatitis C. Magn Reson Med Sci 2012;11:291-297.

32. Shi Y, Guo Q, Xia F, et al. MR elastography for the assessment of hepatic fibrosis in patients with chronic hepatitis B infection: does histologic necroinflammation influence the measurement of hepatic stiffness? Radiology 2014;273:88-98.

33. Castera L. Noninvasive assessment of liver fibrosis. Dig Dis 2015;33:498-503.

34. Sandrin L, Fourquet B, Hasquenoph JM, et al. Transient elastography: a new noninvasive method for assessment of hepatic fibrosis. Ultrasound Med Biol 2003;29:1705-1713.

35. Castéra L, Vergniol J, Foucher J, et al. Prospective comparison of transient elastography, Fibrotest, APRI, and liver biopsy for the assessment of fibrosis in chronic hepatitis C. Gastroenterology 2005;128:343-350.

36. Myers RP, Pomier-Layrargues G, Kirsch R, et al. Feasibility and diagnostic performance of the FibroScan XL probe for liver stiffness measurement in overweight and obese patients. Hepatology 2012;55:199-208

37. Foucher J, Castera L, Bernard PH, et al. Prevalence and factors associated with failure of liver stiffness measurement using Fi-
broScan in a prospective study of 2114 examinations. Eur J Gastroenterol Hepatol 2006;18:411-412.

38. Cui XW, Friedrich-Rust M, De Molo C, Ignee A, Schreiber-Dietrich D, Dietrich CF. Liver elastography, comments on EFSUMB elastography guidelines 2013. World J Gastroenterol 2013;19:63296347.

39. Tang A, Cloutier G, Szeverenyi NM, Sirlin CB. Ultrasound elastography and MR elastography for assessing liver fibrosis: part 2, diagnostic performance, confounders, and future directions. AJR Am J Roentgenol 2015;205:33-40.

40. Perumpail RB, Levitsky J, Wang Y, et al. MRI-guided biopsy to correlate tissue specimens with MR elastography stiffness readings in liver transplants. Acad Radiol 2012;19:1121-1126.

41. Kamphues C, Klatt D, Bova R, et al. Viscoelasticity-based magnetic resonance elastography for the assessment of liver fibrosis in hepatitis C patients after liver transplantation. Rofo 2012;184:1013-1019.

42. Talwalkar J, Yin M, Venkatesh S, et al. Feasibility of in vivo MR elastographic splenic stiffness measurements in the assessment of portal hypertension. AJR Am J Roentgenol 2009;193:122-127.

43. Ronot M, Lambert S, Elkrief L, et al. Assessment of portal hypertension and high-risk oesophageal varices with liver and spleen three-dimensional multifrequency MR elastography in liver cirrhosis. Eur Radiol 2014;24:1394-1402.

44. Nedredal GI, Yin M, McKenzie T, et al. Portal hypertension correlates with splenic stiffness as measured with MR elastography. J Magn Reson Imaging 2011;34:79-87.

45. Low G, Owen NE, Joubert I, et al. Magnetic resonance elastography in the detection of hepatorenal syndrome in patients with cirrhosis and ascites. Eur Radiol 2015;25:2851-2858.

46. Adams LA, Lymp JF, St Sauver J, et al. The natural history of nonalcoholic fatty liver disease: a population-based cohort study. Gastroenterology 2005;129:113-121.

47. Chalasani N, Younossi Z, Lavine JE, et al. The diagnosis and management of non-alcoholic fatty liver disease: practice guideline by the American Gastroenterological Association, American Association for the Study of Liver Diseases, and American College of Gastroenterology. Gastroenterology 2012;142:1592-1609.

48. Ratziu V, Charlotte F, Heurtier A, et al. Sampling variability of liver biopsy in nonalcoholic fatty liver disease. Gastroenterology 2005;128:1898-1906.

49. Lee SS, Park SH, Kim HJ, et al. Non-invasive assessment of hepatic steatosis: prospective comparison of the accuracy of imaging examinations. J Hepatol 2010;52:579-585.

50. van Werven JR, Marsman HA, Nederveen AJ, et al. Assessment of hepatic steatosis in patients undergoing liver resection: comparison of US, CT, T1-weighted dual-echo MR imaging, and pointresolved 1H MR spectroscopy. Radiology 2010;256:159-168.

51. Hepburn MJ, Vos JA, Fillman EP, Lawitz EJ. The accuracy of the report of hepatic steatosis on ultrasonography in patients infected with hepatitis $\mathrm{C}$ in a clinical setting: a retrospective observational study. BMC Gastroenterol 2005;5:14. 
52. Strauss S, Gavish E, Gottlieb P, Katsnelson L. Interobserver and intraobserver variability in the sonographic assessment of fatty liver. AJR Am J Roentgenol 2007;189:W320-W323.

53. Artz NS, Haufe WM, Hooker CA, et al. Reproducibility of MRbased liver fat quantification across field strength: same-day comparison between 1.5T and 3T in obese subjects. J Magn Reson Imaging 2015;42:811-817.

54. Noureddin M, Lam J, Peterson MR, et al. Utility of magnetic resonance imaging versus histology for quantifying changes in liver fat in nonalcoholic fatty liver disease trials. Hepatology 2013;58:1930-1940.

55. Dong Z, Luo Y, Zhang Z, et al. MR quantification of total liver fat in patients with impaired glucose tolerance and healthy subjects. PloS One 2014;9:e111283.

56. Tang A, Rabasa-Lhoret R, Castel H, et al. Effects of insulin glargine and liraglutide therapy on liver fat as measured by magnetic resonance in patients with type 2 diabetes: a randomized trial. Diabetes Care 2015;38:1339-1346.

57. Patel NS, Doycheva I, Peterson MR, et al. Effect of weight loss on magnetic resonance imaging estimation of liver fat and volume in patients with nonalcoholic steatohepatitis. Clin Gastroenterol Hepatol 2015;13:561-568.e1.

58. Idilman IS, Keskin 0, Elhan AH, Idilman R, Karcaaltincaba M. Impact of sequential proton density fat fraction for quantification of hepatic steatosis in nonalcoholic fatty liver disease. Scand J Gastroenterol 2014;49:617-624.

59. Kang BK, Yu ES, Lee SS, et al. Hepatic fat quantification: a prospective comparison of magnetic resonance spectroscopy and analysis methods for chemical-shift resonance imaging with histologic assessment as the reference standard. Invest Radiol 2012;47:368-375.

60. Reeder SB, Cruite I, Hamilton G, Sirlin CB. Quantitative assessment of liver fat with magnetic resonance imaging and spectroscopy. J Magn Reson Imaging 2011;34:729-749.

61. Lee SS, Lee Y, Kim N, et al. Hepatic fat quantification using chemical shift MR imaging and MR spectroscopy in the presence of hepatic iron deposition: validation in phantoms and in patients with chronic liver disease. J Magn Reson Imaging 2011;33:13901398.

62. Yokoo T, Bydder M, Hamilton G, et al. Nonalcoholic fatty liver disease: diagnostic and fat-grading accuracy of low-flip-angle multiecho gradient-recalled-echo MR imaging at $1.5 \mathrm{~T}$. Radiology 2009;251:67-76.

63. Yu H, Shimakawa A, Hines CD, et al. Combination of complexbased and magnitude-based multiecho water-fat separation for accurate quantification of fat-fraction. Magn Reson Med 2011;66:199-206.

64. Meisamy S, Hines CD, Hamilton G, et al. Quantification of hepatic steatosis with T1-independent, T2-corrected MR imaging with spectral modeling of fat: blinded comparison with MR spectroscopy. Radiology 2011;258:767-775.

65. Kukuk GM, Hittatiya K, Sprinkart AM, et al. Comparison between modified Dixon MRI techniques, MR spectroscopic relaxometry, and different histologic quantification methods in the assessment of hepatic steatosis. Eur Radiol 2015;25:2869-2879.

66. Brittenham GM, Badman DG. Noninvasive measurement of iron: report of an NIDDK workshop. Blood 2003;101:15-19.

67. Fujita N, Sugimoto R, Urawa N, et al. Hepatic iron accumulation is associated with disease progression and resistance to interferon/ribavirin combination therapy in chronic hepatitis C. J Gastroenterol Hepatol 2007;22:1886-1893.

68. Allen KJ, Gurrin LC, Constantine CC, et al. Iron-overload-related disease in HFE hereditary hemochromatosis. N Engl J Med 2008;358:221-230.

69. Rowe JW, Wands JR, Mezey E, et al. Familial hemochromatosis: characteristics of the precirrhotic stage in a large kindred. Medicine 1977;56:197-211.

70. Barry M, Sherlock S. Measurement of liver-iron concentration in needle-biopsy specimens. Lancet 1971;1:100-103.

71. Villeneuve JP, Bilodeau M, Lepage R, Cote J, Lefebvre M. Variability in hepatic iron concentration measurement from needlebiopsy specimens. J Hepatol 1996;25:172-177.

72. Papakonstantinou 0, Kostaridou S, Maris T, et al. Quantification of liver iron overload by $\mathrm{T} 2$ quantitative magnetic resonance imaging in thalassemia: impact of chronic hepatitis $C$ on measurements. J Pediatr Hematol Oncol 1999;21:142-148.

73. Sirlin CB, Reeder SB. Magnetic resonance imaging quantification of liver iron. Magn Reson Imaging Clin N Am 2010;18:359-381.

74. Alustiza Echeverria JM, Castiella A, Emparanza JI. Quantification of iron concentration in the liver by MRI. Insights Imaging 2012;3:173-180.

75. Westwood M, Anderson LJ, Firmin DN, et al. A single breathhold multiecho $\mathrm{T}^{*}$ cardiovascular magnetic resonance technique for diagnosis of myocardial iron overload. J Magn Reson Imaging 2003;18:33-39.

76. Wood JC. Magnetic resonance imaging measurement of iron overload. Curr Opin Hematol 2007;14:183-190.

77. Gandon Y, Olivie D, Guyader D, et al. Non-invasive assessment of hepatic iron stores by MRI. Lancet 2004;363:357-362.

78. Castiella A, Alústiza JM, Emparanza JI, Zapata EM, Costero B, Díez MI. Liver iron concentration quantification by MRI: are recommended protocols accurate enough for clinical practice? Eur Radiol 2011;21:137-141.

79. Clément O, Mühler A, Vexler V, Berthezène Y, Brasch RC. Gadolinium-ethoxybenzyl-DTPA, a new liver-specific magnetic resonance contrast agent: kinetic and enhancement patterns in normal and cholestatic rats. Invest Radiol 1992;27:612-619.

80. Shimizu J, Dono K, Gotoh M, et al. Evaluation of regional liver function by gadolinium-EOB-DTPA-enhanced MR imaging. Dig Dis Sci 1999;44:1330-1337.

81. Kim T, Murakami T, Hasuike Y, et al. Experimental hepatic dysfunction: evaluation by MRI with Gd-EOB-DTPA. J Magn Reson Imaging 1997;7:683-688.

82. Van Beers BE, Pastor CM, Hussain HK. Primovist, eovist: what to 
expect? J Hepatol 2012;57:421-429.

83. Tsuda N, Okada M, Murakami T. Potential of gadolinium-ethoxybenzyl-diethylenetriamine pentaacetic acid (Gd-EOB-DTPA) for differential diagnosis of nonalcoholic steatohepatitis and fatty liver in rats using magnetic resonance imaging. Invest Radiol 2007;42:242-247.

84. Leonhardt M, Keiser M, Oswald S, et al. Hepatic uptake of the magnetic resonance imaging contrast agent Gd-EOB-DTPA: role of human organic anion transporters. Drug Metab Dispos 2010;38:1024-1028.

85. Verloh N, Haimerl M, Zeman F, et al. Assessing liver function by liver enhancement during the hepatobiliary phase with Gd-EOBDTPA-enhanced MRI at 3 Tesla. Eur Radiol 2014;24:1013-1019.

86. Nilsson H, Blomqvist L, Douglas L, Nordell A, Jonas E. Assessment of liver function in primary biliary cirrhosis using Gd-EOBDTPA-enhanced liver MRI. HPB (Oxford) 2010;12:567-576.

87. Higaki A, Tamada T, Sone T, et al. Potential clinical factors affecting hepatobiliary enhancement at Gd-EOB-DTPA-enhanced MR imaging. Magn Reson Imaging 2012;30:689-693.

88. Nakamura S, Awai K, Utsunomiya D, et al. Chronological evaluation of liver enhancement in patients with chronic liver disease at Gd-EOB-DTPA-enhanced 3-T MR imaging: does liver function correlate with enhancement? Jpn J Radiol 2012;30:25-33.

89. Utsunomiya T, Shimada M, Hanaoka J, et al. Possible utility of MRI using Gd-EOB-DTPA for estimating liver functional reserve. J Gastroenterol 2012;47:470-476.

90. Nilsson H, Karlgren S, Blomqvist L, Jonas E. The inhomogeneous distribution of liver function: possible impact on the prediction of post-operative remnant liver function. HPB (Oxford) 2015;17:272-277.

91. Kanki A, Tamada T, Higaki A, et al. Hepatic parenchymal enhancement at Gd-EOB-DTPA-enhanced MR imaging: correlation with morphological grading of severity in cirrhosis and chronic hepatitis. Magn Reson Imaging 2012;30:356-360.

92. Katsube T, Okada M, Kumano S, et al. Estimation of liver function using T1 mapping on Gd-EOB-DTPA-enhanced magnetic resonance imaging. Invest Radiol 2011;46:277-283.

93. Feier D, Balassy C, Bastati N, Stift J, Badea R, Ba-Ssalamah A.
Liver fibrosis: histopathologic and biochemical influences on diagnostic efficacy of hepatobiliary contrast-enhanced MR imaging in staging. Radiology 2013;269:460-468.

94. Kato N, Yokawa T, Tamura A, Heshiki A, Ebert W, Weinmann HJ. Gadolinium-ethoxybenzyl-diethylenetriamine-pentaacetic acid interaction with clinical drugs in rats. Invest Radiol 2002;37:680684.

95. Nassif A, Jia J, Keiser M, et al. Visualization of hepatic uptake transporter function in healthy subjects by using gadoxetic acidenhanced MR imaging. Radiology 2012;264:741-750.

96. Sandrasegaran K. Functional MR imaging of the abdomen. Radiol Clin North Am 2014;52:883-903.

97. Varenika V, Fu Y, Maher JJ, et al. Hepatic fibrosis: evaluation with semiquantitative contrast-enhanced CT. Radiology 2013;266:151-158.

98. Rosenkrantz AB, Storey P, Gilet AG, et al. Magnetization transfer contrast-prepared MR imaging of the liver: inability to distinguish healthy from cirrhotic liver. Radiology 2012;262:136-143.

99. Allkemper T, Sagmeister F, Cicinnati V, et al. Evaluation of fibrotic liver disease with whole-liver T1rho MR imaging: a feasibility study at 1.5 T. Radiology 2014;271:408-415.

100. Rasucher I, Eiber M, Ganter C, et al. Evaluation of T1rho as a potential MR biomarker for liver cirrhosis: comparison of healthy control subjects and patients with liver cirrhosis. Eur J Radiol 2014;83:900-904.

101. Deng M, Zhao F, Yuan J, Ahuja AT, Wang YX. Liver T1 $\rho$ MRI measurement in healthy human subjects at $3 \mathrm{~T}$ : a preliminary study with a two-dimensional fast-field echo sequence. Br J Radiol 2012;85:e590-e595.

102. Balarsy C, Feier D, Peck-Radosavljevic M, et al. Susceptibilityweighted MR imaging in the grading of liver fibrosis: a feasibility study. Radiology 2014;270:149-158.

103. Yoon JH, Lee JM, Suh KS, et al. Combined use of MR Fat quantification and MR elastography in living liver donors: can it reduce the need for preoperative liver biopsy? Radiology 2015;276:453464.

104. Venkatesh SK, Ehman RL. Magnetic resonance elastography of liver. Magn Reson Imaging Clin N Am 2014;22:433-446. 